\title{
Review on RF Tunable Filters
}

\author{
K.Thilagavathi \\ Department of Electronics and Communication Engineering \\ Kumaraguru College of Technology, Coimbatore, India. \\ M. Balakumar \\ Department of Electronics and Communication Engineering \\ Kumaraguru College of Technology, Coimbatore, India.
}

\begin{abstract}
Multi-band radios are necessary nowadays to provide optimal data rates in a network with a varied and greasy landscape of coverage areas (3G, HSPA, LTE, etc.). As required the number of bands are increased, the total cost of discrete RF filters justifies the use of tunable RF filters. Tunable filters are one of the key to signal chain amalgamation. The main requirements for a tunable filter are high unloaded quality factor, wide tuning range, high tuning speed, high linearity, and small size. Tunable filters play a vital role in many RF receiver applications. These filters are used to separate or combine different frequencies bands because the electromagnetic spectrum is limited and has to be shared. Tunable RF filters are used to select or confine the RF signals within assigned spectral limits. Thanks to the numerous technology and the recent developments in design and implementations of the tunable RF filters, usage of these filters has increased exponentially. Emerging applications such as wireless communications continue to challenge RF/microwave filters with ever more stringent requirements higher performance, smaller size, lighter weight, and lower cost. Depending on the requirements and specifications, RF filters may be designed as lumped element or distributed element circuits. They may be realized in various transmission line structures, such as waveguide, coaxial line, and micro strip. Compared to the other types of filter, a tunable filter has its own place. There are many challenging research problems existing with tunable filters. Beginning with an overview of the tunable filters literature, this paper compares and discuss about the various tunable solutions and their issues with different types of tuning. High performance filters necessitate high resonator quality factors. Although filters are traditionally treated as passive devices, tunable filters need to be treated as active devices. Tuning elements invariably introduce non-linearities that limit the useful power handling of the tunable filter.
\end{abstract}

Keywords: Tunable RF filters; dual-behaviour resonators; micro strip, microwave filters, planar filters, coupling matrix.

\section{INTRODUCTION}

Multiband radios are a topic of significant recent interest due to increased demand for smartphones with global coverage and the bare allocation of wireless spectrum for mobile applications. The wireless industry has a fast growth, various RF technologies have been developed. Cellular technology standards (GSM, EDGE, 3G, etc.) for long-range voice, data, video transfer and wireless local area network standards for home and office area networks are some major applications. This has motivated the industry to look for multiband devices to enable anywhere anytime connectivity. The ultimate solution is to use software-defined radio (SDR).

Tunable filters are aimed at addressing the multiband problem by permitting a single component to handle multiple bands so as to combine multiple frontend signal chains into one. A single tunable analog signal chain with software defined baseband processing has the potential to reduce circuit area and result in more flexible and scalable multiband radios. Tunable filters promise to enable frequency agile receivers and have been implemented using a variety of tuning elements including varactor diodes, radio frequency micro-electromechanical systems (RF MEMS), piezoelectric elements and para electric elements. These tunable elements can enable reconfigurable single chip wireless frontends resulting in smaller, more power efficient and less costly multi-standard radios. We first researched ways of tuning the filter's center frequency by investigating the diverse technologies for the implementation of tunable capacitors, varactor diodes, DTC, RF MEMS discussed in Chapter II.

\section{LITERATURE REVIEW}

In this chapter a literature review of relevant material is presented. In Section 2.1 and 2.2, a literature review of tunable filters and filter tuning elements is outlined. Literature on tunable cavity filters, planar filters and tunable components is presented. 
The final review section, Section 2.3, describes a new approach in design of tunable RF filters using dual behaviour resonators (DBR) topology.

\subsection{Tunable Filters and Tuning Elements}

In this section several dfferent tunable planar and cavity filter designs will be highlighted. The section is split into two subsections - one on tunable cavity filters and the other on tunable planar filters. Microwave filters can be constructed using a large array of technologies and are traditionally fabricated with tuning elements to compensate for manufacturing tolerances. The most popular tuning elements for cafittyrs (waveguide, coaxial, dielectric resonator, etc.) are tuning screws [1]. In planar technologies trimming capacitors can be used though it is not uncommon to physically trim sections of micro strip line to achieve the desired tuning ffect [2] or even to swafixed capacitors. These tuning elements and techn iques are applied during assembly and generally not adjusted after the filter leaves the factory.

\subsubsection{Tunable Cavity Filters}

Commercial post-production tunable cavityfilters are adjusted by turning a vernier knob such as the one shown in Figure. 2.1a. The knob is attached to a common shaft from which the tuning elements are coupled via a geared mechanism [3]. The bandwidth is usuallyixed and is not adjustable [4]. The single tuning knob simplifies the use of the filter since it only requires adjusting one element. A picture of the BT series of tunable filters from K\&L Microwave is shown in Figure. 2.1a. the off-the shelf version of the BT series has a 5-section chebyshev response with a constant fractional bandwidth of $5 \%$ but can be customized for a fractional bandwidth from $1 \%$ to $10 \%$.

Commercial filters with tuning knobs for each resonator are also available as shown in Figure. 2.1b [5]. However, these filters require the use of a vector network analyser (VNA) to tune the filter and may require expertise to appropriately tune the $\mathrm{N}$ knobs for an Nth-order filter. Also, even the N-knob filters in [10] do not have direct control over the bandwidth since their $\mathrm{N}$ tuning knobs do ffent axcess to tune the $\mathrm{N}-1$ additional coupling values between the cavities.

Thus far all the tunableilters discussed have required manual tuning. Indeed, most of the commercially available tunable filters of this type do since they are intended for test and measurement, surveillance and niche wireless communications applications. The single vernier knob tunablefilters are also available in motorized versions with electronic general purpose interface bus (GPIB) interfaces [4] but they also do noffer tunable bandwidth. The bandwidth of these mechanically tunablefilters is often fixed rel ative to the center frequency; i.e. their percentage bandwidth iffixed. The absolute bandwidth of this class of filters varies with the center frequency.

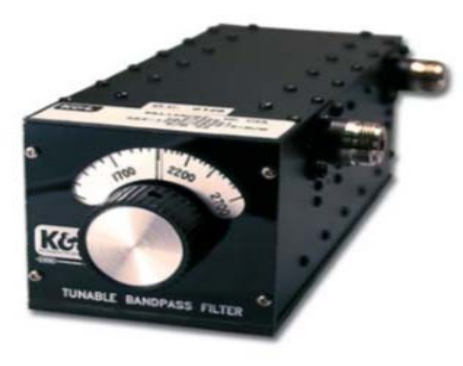

(a) Tunable cavity filter from K\&L

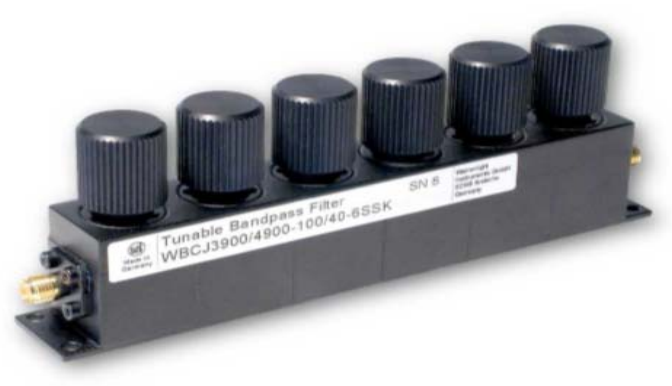

(b) Tunable cavity filter with individual

Microwave with a single vernier knob [4] tuning knobs [5]

Figure 2.1: Tunable cavity filters from K\&L Microwave and Wainwright Instruments GmbH [5]

Electronic tuning of cavityfilters is done by modifying the field within the cavity using an electronic actu ator that disturbs thefields inside the cavity. These actuators can be constructed with simple stepper motors [6] or 
even piezoelectric actuators as shown in the dual mode dielectric resonator tunablfilter shown in Figure. 2.2 [7].

A piezoelectric tuning mechanism was also used in a series of works on highly loaded evanescent coupled cavities. Initial designs used piezoelectric actuators that deformed a copper-clad circuit board membrane that was soldered on top of a low temperature co-fired ceramic (LTCC) circuit [8]. Subsequent designs were based on a substrate integrated technique.

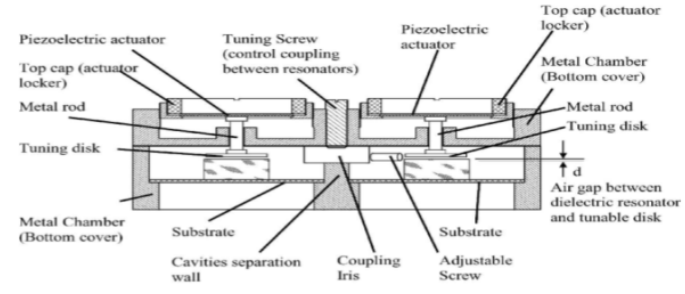

(a) Lateral view

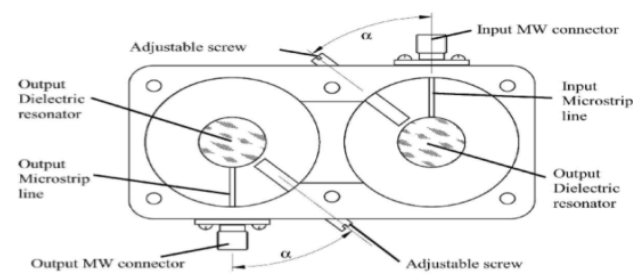

(b) Top view

Figure 2.2: Piezoelectric actuator tuned dual mode resonator 4-pole filter [7]

The resonators have been studied in multiple designs including tunable bandwidtfilters [9], triplexer [11], reconfigurable order filters [12] and band pass to band stop switchablfelters [13]. Tuning elements for the evanescent mode cavities based on micro-electro-mechanical system (MEMS) technology using both electrostatic [14] and magneto static actuation [15] have also been demonstrated. The primary advantage of the MEMS implementation is the elimination of hysteresis which can be detrimental to the repeatable tuning of the filter. Ultimately, the work has culminated in thefield programmable filter array (FPFA). Analogous to a field programmable gate array (FPGA) in the digital domain, thefield programmable filter array permits an arbitrary arrangement of coupled resonators to realize fi lters of varying order, transmission zeros, band pass or band stop response [16].

\subsubsection{Tunable Planar Filters}

Tunable cavity filters are easily tuned by mechanical means due to their large size. Inserting an electrically or magnetically visible material into the cavity invariably results in frequency shift. The size of the tuning elements also makes tunable cavity filters more expensive since they require mechanical actuators. Planar filters are more difficult to tune with tuning screws since the electric field tends to be trapped inside the dielectric. The use of tuning screws on planar filters is thus limited to fine tuning during production[17].

Planar filter technology is highly attractive for tunable filters due to its relatively small size and ease of integration with active components. Planar fabrication also results in lower cost and is amenable to mass production. Variable capacitors are the most common tuning element in printed and integrated circuit technology. Electronically tuned variable capacitors can be implemented using several dferent technologies. Semiconductor varactors are the most common [19-20] with significant research attention in MEMS [21 -23] and barium strontium titanate (BST) tunable capacitors [24-25]. The trade-offs between the three are many including cost, linearity (intermodulation distortion [18]), Q-factor and tuning range. MEMS technology is the most promising overall but suffers from substantial manufacturing challenges that have thus far resulted in high costs. BST is particularly attractive since it has improved reliability and reduced packaging require- ments. Commercial availability of BST materials is still fairly limited. Semiconductor varactors are common but have relatively low Q-factors at higher frequencies and have lower linearity than MEMS and BST.

\subsection{Tuning Elements}

A set of various tuning element that have been utilized to design tunable filters throughout the history of engineering. The device that used for tuning the filter includes Yttrium Iron Garnet ferrimagnet, Barium Strontium Titanate ferroelectric material, varactor diodes, MEMS capacitors and digitally tunable capacitors. For implementation of various tuning element, there are many parameters regarding these technologies that can 
affect the performance of the circuit. Therefore, it is important to consider all these before making a choice on which technology or element to use for a specific application

2.2.1 Yttrium Iron Garnet (YIG) Tunable Filters: One of the oldest technologies for implementing filter tuning is the Yttrium Iron Garnet technology. Yttrium Iron Garnet is a ferrimagnetic material whose magnetic dipoles process at a frequency of the external magnetic field applied to the material. Additionally, this technology exhibits extremely low losses, which attributes to YIG being a good candidate for tunable filter technology. Phillip S. Carter began exploring this tuning element in the 1960s and was one of the first researchers to investigate and explain its concept of operation. In his article from 1961, "Magnetically-Tunable Microwave Filters Using Single-Crystal Yttrium-Iron-Garnet Resonators" he provides the illustration of concept shown in Figure 2.3. The two coils are perpendicular to each other and a small sample of YIG ferrite is placed at the center of their cross section, as shown in Figure 2.3. Once the DC magnetic field is applied along the z-axis and a RF driven current Ix*ejwt (note the frequency dependency) is provided at the terminals of the x coil, magnetic dipoles in the YIG ferrite process around the x-axis.

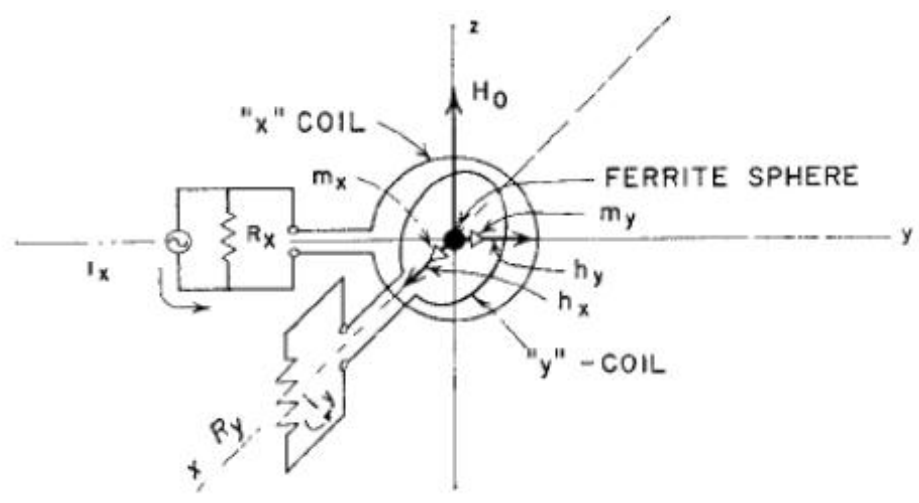

Figure 2.3 Magnetic resonance filter

This induces an RF magnetic moment along the y-axis and voltage in the y circuit. Therefore, the resonant frequency of the YIG filter is tuned by changing the magnetic field and the current that are externally applied to the ferrite. However, there is a requirement that the unloaded Q value of the resonator is high in a low-insertion and narrow band pass filter implementation.

2.2.2 Barium Strontium Titanate Tunable Filter: Barium Strontium Titanate (BST) is a ferroelectric material that is a promising element or technology for the applications of tunable filters. A few ideal characteristics include its dependence on the dielectric permittivity of an applied electric field, high dielectric constant and low production costs. Ferroelectric materials are dielectric materials known for their ability to achieve spontaneous polarization that can be reversed in the presence of an external electric field.

When an external field is applied to a regular dielectric material, the electric dipole moments within the material will align themselves in accordance with the direction of the applied electric field. On the other hand, in materials such as ferroelectrics, this polarization can happen even without the external electric field. Once the electric field that does not exceed the breakdown limit of the crystal is applied to the ferroelectric, its dipole moments will reverse to align with the field. Another unique aspect of the ferroelectric materials is that they become para-electric past the transition temperature, known as the Curie temperature. At the Curie temperature they undergo a structural change while achieving the highest dielectric constant that decreases with further temperature increase in Figure 2.4.

Above the Curie temperature, the material no longer has spontaneous polarization and it obtains para- electric properties. Para electric materials become polarized in the presence of an external electric field just like the ferroelectric materials, but unlike ferroelectrics, polarization in para electrics can occur even when there is no permanent electric dipole in the material. Additionally, polarization of para electric materials returns to zero in the absence of the external field. BST has been found as one of the most suitable ferroelectrics for the tunable filter application as it has a higher dielectric constant, lower losses and higher tunability. Moreover, its high capacitance density allows for construction of higher capacitor values within a smaller area. Unlike conventional 
varactor diodes, BST varactor have no forward conduction region and perform well in applications involving high RF voltage swings over the full range of the DC tuning voltage.

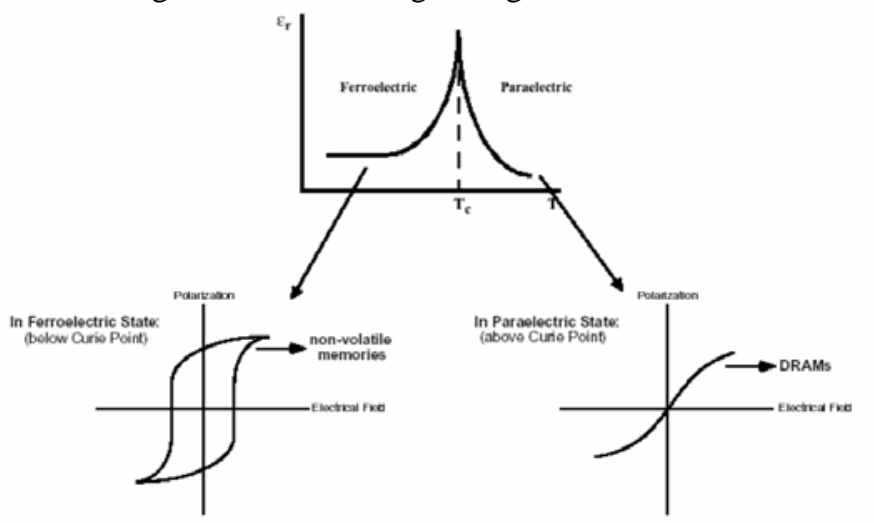

Figure 2.4 Ferroelectric material change of state at Tc

2.2.3 Varactor diodes: Varactor diodes, or varicap diodes, are semiconductor devices whose capacitance varies as a function of the voltage applied across its terminals. They are widely used in applications where voltage controlled variable capacitance is required. Thus, a varactor diode is one of the candidates that can be integrated as part of a tunable filter design approach. Varactor diode is in essence a p-n junction exhibiting the characteristics of a variable capacitor. Under reverse bias condition, no current can flow and the diodes reverse resistance is almost infinity. The depletion region at the p-n junction acts like an insulating dielectric sandwiched between conductive plates of a capacitor. The symbol for the varactor diode can be seen in Figure 2.5 .

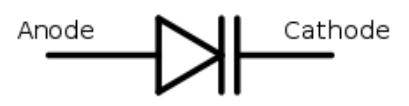

Figure 2.5. Varactor diode symbol

A varactor diode tends to be physically small, inexpensive and has a very high tuning speed, which makes its use advantageous in tunable filters. However, its disadvantages include low Q value, poor power handling capabilities and highly nonlinear characteristics at high frequencies.

2.2.4 Digitally Tunable Capacitor: Digitally Tunable Capacitors (DTCs) are variable circuit components whose capacitance is controlled by a set of FET switches in a combination with a digital SPI interface. The DTCs are made up of several high Q-factor metal-insulator-metal (MIM) capacitors and an array of digitally controlled FET switches that determine the total value of the capacitor bank available at a certain control setting as can be seen in figure 2.6. This control setting is a digital input that feeds in a binary value corresponding to a certain number of switches to be turned on and hence determining how many capacitors will contribute to the total capacitance value

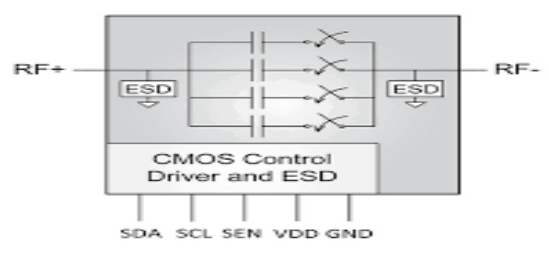

Figure 2.6 Block diagram of DTC

MIM capacitors are generally connected in parallel to each other and each capacitor is connected to a FET switch in series. The maximum capacitance is achieved when all the switches are ON since the total capacitance of individually connected capacitors in parallel is additive. The minimum capacitance is achieved when all the switches are off. In Figure 2.7 is the equivalent circuit for the DTC. It consists of three main parts: The tuning core (RS and CS), the parasitic package inductance (LS) and the shunt parasitic network (CP, RP1, RP2). 


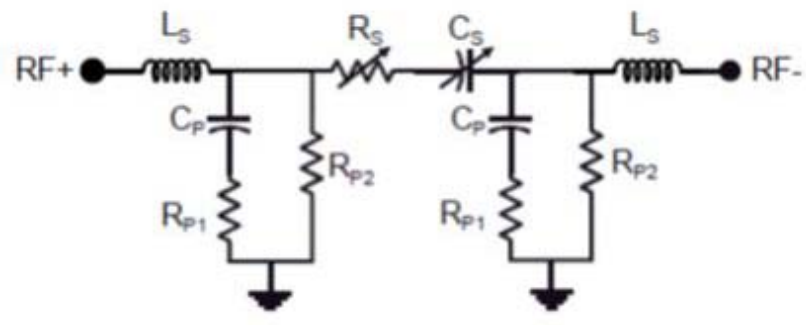

Figure 2.7 Equivalent circuit for DTC 11

One advantage of the DTC is that it has a very linear response. Its capacitance is proportional to input from the digital control circuit in a discrete fashion.

2.2.6 Comparison of Several Component Technologies: Several of the aforementioned technologies available for tunable microwave applications are compared in table 2.1.

\begin{tabular}{|c|c|c|c|c|c|}
\hline Properties & $\begin{array}{c}\text { Varactor } \\
\text { Diode }\end{array}$ & MEMS & BST & DTC & YIG \\
\hline $\begin{array}{c}\text { Tumability } \\
\text { (High Q) }\end{array}$ & Good & Low & Good & High & High \\
\hline RF loss & $\begin{array}{c}\text { Moderate } \\
(\mathrm{Q}<60 \\
\text { typically })\end{array}$ & $\begin{array}{c}\text { Very Good } \\
(\mathrm{Q}<200)\end{array}$ & $\begin{array}{c}\text { Moderate } \\
(\mathrm{Q}<100 \\
\text { typically })\end{array}$ & $\begin{array}{c}\text { Moderate } \\
(\mathrm{Q}<50 \\
\text { typically })\end{array}$ & $\begin{array}{c}\text { Very good } \\
(\mathrm{Q}<200)\end{array}$ \\
\hline $\begin{array}{c}\text { Control } \\
\text { Voltage }\end{array}$ & $<10 \mathrm{~V}$ & $<60 \mathrm{~V}$ & $<5-30 \mathrm{~V}$ & $<30 \mathrm{~V}$ & $<28 \mathrm{~V}$ \\
\hline $\begin{array}{c}\text { Tuning } \\
\text { Speed }\end{array}$ & $\begin{array}{c}\text { Fast } \\
1-5\end{array}$ & $\begin{array}{c}\text { Slow } \\
\text { nanoseconds }\end{array}$ & $\begin{array}{c}\text { Fast } \\
\text { microseconds }\end{array}$ & $\begin{array}{c}\text { Fast } \\
<12 \\
\text { nanoseconds }\end{array}$ & $\begin{array}{c}\text { Slow } \\
\text { nanoseconds } \\
\text { milliseconds }\end{array}$ \\
$\begin{array}{c}\text { Power } \\
\text { Handling } \\
\text { Capability }\end{array}$ & Poor & Excellent & $\begin{array}{c}\text { Trades with } \\
\text { control } \\
\text { voltage }\end{array}$ & Excellent & Excellent \\
\hline
\end{tabular}

Table 2.1.Comparison of competing technologies

According to Table 2.1, varactor diodes have good tunability, fast tuning speed and small size. However, these components have junction noise, poor power handling capability and require a reverse bias condition to sustain a capacitive effect. MEMS based devices are linear in response as well as maintain very low RF loss high tunability properties. Nevertheless, these devices are relatively slow, can be unreliable and have high packaging cost. BST technology is advantageous over both semiconductor and MEMS devices due to low cost, higher break down voltages, higher power handling capability and low packaging cost. YIG materials excel in all factors except in the tuning speed as they exhibits speeds in the range of milliseconds. Finally, the DTCs have high tenability, moderate RF loss, operate as high speeds and have excellent power handling capability.

\subsection{Tunable Filter Design}

\subsubsection{Tunable Filter Design using DBR topology}

2.3.1.1 Dual-Behaviour Resonator: The use of dual-behaviour resonators (DBRs) appears as a quite convenient solution because they allow the control of two attenuated bands on either side of one band pass [19]. A DBR results from the association of two different parallel band stop structures. Each of them brings its own transmission zero with respect to its fundamental resonant condition. At the same time, their association is transparent within a given operating frequency once the band stop structures have been properly connected under constructive recombination criteria. This results in a band pass response created between the above mentioned lower and upper rejected bands. According to the number of available parameters and to the initial behaviour of each band stop structure, DBR allows an independent control of the following: one pole in the operating bandwidth, one transmission zero in the lower attenuated band, one transmission zero in the upper attenuated band.

Some possible topologies based on the DBR concept. All of them allow the independent control of one band pass and two band stops. The first part focuses on the use of stepped-impedance open-circuited stubs. General 
equations about this structure have already been introduced, but the proposed simplification induces the dependence of the upper and lower transmission zeros [20]. The simplification of the equations proposed here keeps four physical parameters independent. Thus, through this new synthesis four electrical parameters, i.e., the central frequency, bandwidth, and two transmission zeros, are independently controlled.

2.3.1.2 DBR Topology Overview: DBR topology is based on the parallel association of two stubs which gives a band pass response. It has a very narrow band pass response and $2 \mathrm{n}$ transmission zeros ( $\mathrm{n}$ low and $\mathrm{n}$ high), with $\mathrm{n}$ is the filter order. Figure.2.8 shows a third order DBR filter. This topology permits to control central frequency and bandwidth independently, but it has an inconvenience which is the spurious resonances at low and high frequencies. This problem can be solved using the Capacitive-Coupled Dual Behaviour resonators.

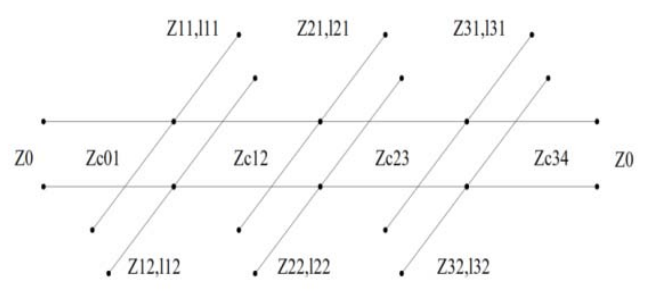

Figure 2.8 Third order DBR filter topology

For controlling central frequency and bandwidth independently, varicap diodes are inserted at the end of each stub as shown in figure.2.9. Varicaps are modelled by ideal variable capacitances. CT are the varicaps placed at the end of the stubs providing the low transmission zeros and CD are the varicaps placed at the end of the stubs providing the high transmission zeros.

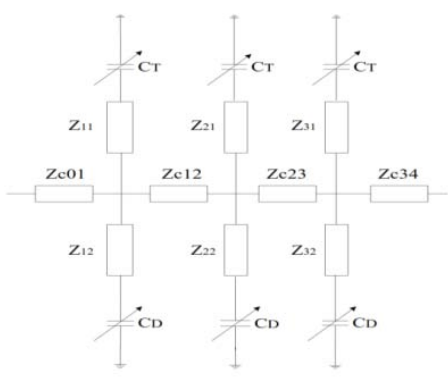

Figure 2.9. Third order tunable DBR structure

2.3.1.3 Design Methodology: In this section, we propose a methodology for the design of tunable RF filters using DBR topology. This algorithm could be an aided-design tool used by RF filter engineers and designers. Having design specifications for each elementary RF filter, tunable RF filter design steps are as follows.

\section{Step1: RF filters specifications and design choice.}

In this section, we get the specifications parameters for filter synthesis from the filters templates of the standards which are essentially the pass band edge frequencies (Fp1i, Fp2i), the rejection frequencies (Fs1i, Fs2i) and their associated attenuation (Asi). fci is the center frequency for ith RF band. RF filter technology and topology have to be chosen by RF designers before going to implementation. In our case, DBR topology and microstrip technology are selected to develop a tunable RF filter.

$$
\mathrm{f}_{\mathrm{ci}}=\sqrt{\mathrm{F}_{\mathrm{p} 1 \mathrm{i}} \cdot \mathrm{F}_{\mathrm{p} 2 \mathrm{i}}}
$$

Step 2: Order synthesis

Choose the appropriate approximation to filter design such as Chebyshev, Butterworth, Elliptic approximations [23]. The choice is made when considering reduced filter order and acceptable response linearity. Then synthesize the order ni for each elementary filter specifications [23].

Step 3: Physical topology synthesis 
Tunable filter order $\mathrm{m}$ is given by :

$\mathrm{m}=\max _{1<i<\mathrm{N}} \mathrm{n}_{\mathrm{i}}$

Use $\mathrm{m}$, the pass band edge frequencies $\left(\mathrm{F}_{\mathrm{p} 1 \mathrm{~N}}, \mathrm{~F}_{\mathrm{p} 2 \mathrm{~N}}\right)$ and the rejection frequencies $\left(\mathrm{F}_{\mathrm{s} 1 \mathrm{~N}}, \mathrm{~F}_{\mathrm{s} 2 \mathrm{~N}}\right)$ to synthesize $\mathrm{RF}$ filter physical structure based on DBR topology [20].

Step 4: Reconfigurable elements synthesis

Set $C_{T N}$ and $C_{D N}$ to $C_{0}=0$ in order to fit Nth RF filter specifications, where $C_{0}$ is an intermediate parameter used for center frequency control. For iteration $k$, increase first $\mathrm{C}_{0}$ in order to allow response filter moving around $\mathrm{f}_{\mathrm{ck}}$ center frequency, where $\mathrm{C}_{\mathrm{Tk}}=\mathrm{C}_{\mathrm{Dk}}=\mathrm{C}_{0}$. To increase filter bandwidth, $\mathrm{C}_{\mathrm{Tk}}$ has to be increased and vice versa.

$$
\mathrm{C}_{\mathrm{Dk}}=2 \mathrm{C}_{0}-\mathrm{C}_{\mathrm{Tk}}
$$

$\mathrm{C}_{\mathrm{Tk}}$ and $\mathrm{C}_{\mathrm{Dk}}$ are algorithm outputs needed for the design of tunable RF filter using DBR topology.

\subsubsection{APPLICATIONS}

\section{SDR RECEIVER ARCHITECTURE OVERVIEW}

As the trend in wireless communication systems today is toward multi-functionality, multiband, large scale integration, miniaturization and low cost, SDR receivers will require wideband or tunable RF front-end modules (Antenna, Band pass Filter, etc.).

Among these modules, RF filters have a most significant role since they affect the receiver performances. The use of wideband RF filter may worsen the performances and may impose some restrictions on the other modules of the receiver (low noise amplifier (LNA), mixers, etc.) due to their high dynamic range[22]..The use of parallel filters with limited frequency bands is more attractive solution for multi-standard SDR receivers but still suffer from important insertion loss and circuit size. The ultimate solution is to replace these filters by one tunable RF filter.

\section{CONCLUSIONS}

In this paper, a brief overview of RF tunable filter is presented. With over a decade of extensive research, there has been a tremendous development and application activities in the RF tunable filter domain. The primary goal of this project was to research, design, and simulate a tunable filter topology and extensive background research into tunable components, and general design issues with filter technology were conducted. It is impossible to give a complete coverage on this topic with limited space and knowledge. There are many challenging research problems facing tunable filter design implementation such as tunable components solutions, and general design issues with filter technology. To improve the design topology of the tunable filter, new methodology to design tunable RF filter with DBR topology has been developed. Then we apply it to design an RF filter for UMTS/WiFi/LTE standards. However, in-depth research is still required on several critical issues so that there can be developments in leaps and bounds in the tunable filter technology.

\section{REFERENCES}

[1] Tuning elements. Johanson Manufacturing. [Online]. Available:http://www. johansonmfg.com/pdf/Tuning-Elements.pdf

[2] G. Tsuzuki, M. P. Hernandez, and B. A. Willemsen, ITuning fork fiter design for hand scribe tuning," in Proc. IEEE MTT-S Int. Microw. Symp. Digest, $12\{17$ June 2005, p. 4pp. 
[3] On line catalogue. RS Microwave Company, Inc. 22 Park Place, PO Box 273, Butler, NJ 07405. [Online]. Available:http://www.rsmicro.com/catalog. htm

[4] BT Series - Tunable Bandpass Filters. K\&L Microwave. [Online]. Available: $\quad$ http://www.klmicrowave.com/productattach/0/ plk196 1 BTSeriesNEW.pdf

[5] Tunable Bandpass Filter. Wainwright Available:http://www.wainwrightfiters.com/pdf/bandpass/tunable/bcj-3900-4900-100-40-6-tun.pdf

[6] B. Yassini, M. Yu, D. Smith, and S. Kellett, \A Ku-band high-Q tunable fiter with stable tuning response,"IEEE Trans. Microw. Theory Tech., vol. 57, no. 12, pp.2948\{2957, Dec. 2009, doi: 10.1109/TMTT.2009.2034215.

[7] N. Alford, O. Buslov, V. Keis, I. Kotelnikov, A. Kozyrev, P. Kulic, and P. Petrov, ITunable 4-pole piezoelectric fiter based on two dielectric resonators," Integrated Ferroelectrics.

[8] H. Joshi, H. H. Sigmarsson, D. Peroulis, and W. J. Chappell, \Highly loaded evanescent cavities for widely tunable high-q fiters," in Proc. IEEE/MTT-S Int. Microwave Symp, 2007, pp. $2133\{2136$.

[9] H. Joshi, H. H. Sigmarsson, S. Moon, D. Peroulis, and W. J. Chappell, \High-q fully reconfigurable tunable bandpass fiters," IEEE Trans. Microw. Theory Tech., vol. 57, no. 12, pp. 3525\{3533, 2009,doi: 10.1109/TMTT.2009.2034309.

[10] S. Moon, H. H. Sigmarsson, H. Joshi, and W. J. Chappell, ISubstrate integrated evanescent-mode cavity fiter tuning ratio," IEEE Microw. Wireless Compon. Lett, vol. 20, no. 8, pp. 450\{452, 2010,doi: 10.1109/LMWC.2010.2050680.

[11] H. Joshi, H. H. Sigmarsson, S. Moon, D. Peroulis, and W. J. Chappell, \Tunable high q narrow-band triplexer," in Proc. IEEE MTT-S Int. Microwave Symp. Digest MTT '09, 2009, pp. 1477\{1480.

[12] H. H. Sigmarsson, J. Lee, D. Peroulis, and W. J. Chappell, \Reconfigurable-order bandpass fiter for frequency agile systems," in Proc. IEEE MTT-S Int. Microwave Symp. Digest (MTT), 2010, pp. 1756\{1759.

[13] E. J. Naglich, J. Lee, D. Peroulis, and W. J. Chappell, \A tunable bandpass-tobandstop reconfigurable fiter with independent bandwidths and tunable response shape," IEEE Trans. Microw. Theory Tech., vol. 58, no. $\quad 12, \quad$ pp. $3770\{3779, \quad 2010, \quad$ doi: 10.1109/TMTT.2010.2086533.

[14] X. Liu, L. P. B. Katehi, W. J. Chappell, and D. Peroulis, \High- img src="/images/tex/228.gif" alt="q"> tunable microwave cavity resonators and fiters using soi-based RF mems tuners," J. Microelectromech. Syst., vol. $\quad 19$, no. 4, pp. 774\{784, 2010,doi: 10.1109/JMEMS.2010.2055544.

[15] M. S. Arif, W. Irshad, X. Liu, W. J. Chappell, and D. Peroulis, \A high-q magnetostatically-tunable all-silicon cavity resonator," in Proc. IEEE MTT-S Int. Microwave Symp. Digest (MTT), 2011, pp. $1\{4$.

[16] J. Lee, E. J. Naglich, H. H. Sigmarsson, D. Peroulis, and W. J. Chappell, \Frequency-agile field-programmable array (fpfa) with multiple functionalities," in Proc. IEEE MTT-S Int. Microwave Symp. Digest (MTT),

H. Peng, X. Zhang, X. Guo, L. Mu, B. Wei, B. Gao, and B. Cao, \Mechanism study of mechanical tuning in fiter
2011, pp. $1\{4$. fiter application," Microw. Opt. Tech. Lett., vol. 49, no. 7, pp. 1565\{1568, 2007. [Online]. Available: http://dx.doi.org/10.1002/mop.22561

[18] M.-T. Nguyen, W. D. Yan, and E. P. W. Horne, \Broadband tunable Filters using high Q passive tunable ICs," Proc. IEEE MTT-S Int. Microw. Symp. Digest, 15\{20 June 2008, pp. $951\{954$.

[19] C. Quendo, E. Rius, and C. Person, "Narrow bandpass filters using dual behaviour resonators,” IEEE Trans. Theory Tech., vol. 51, pp.734-743, Mar. 2003.

[20] J.-T. Kuo, M.-J. Maa, and P.-H. Lu, "A microstrip elliptic function filter with compact miniaturized hairpin resonators," IEEE Microwave Guided Wave Lett., vol. 10, pp. 94-95, Mar. 2000.

[21] K. Wada and O. Hashimoto, "Fundamentals of open-ended resonators and their application to microwave filters," IEICE Trans. Electron., vol.E83-C, no. 11, pp. 1763-1775, Nov. 2000.

[22] V. J. Arkesteijn, E. A.M. Klumperink and B. Nauta, -An AnalogueFront-End Architecture for Software Defined Rađlio, 13th Workshop onCircuits, Systems and Signal Processing, 28-29 November 2002,Veldhoven, the Netherlands, pp.165-168.

[23] J. S. Hong and M. J. Lancaster, -Microstrip Filters for RF/Microwave Applications, „ Wiley Series in Microwave and ISBN 0471-22161-9, Published: 2011, pp.1-471. 